\title{
Enhancement of Lamb Wave Imaging Resolution by Step Pulse Excitation and Prewarping
}

\author{
Shangchen Fu, Lihua Shi, Yinghui Zhou, and Zhikai Fu \\ National Key Laboratory on Electromagnetic Environmental Effects and Electro-Optical Engineering, \\ PLA University of Science and Technology, Nanjing, Jiangsu 210007, China
}

Correspondence should be addressed to Lihua Shi; shilhnj@163.com

Received 13 August 2014; Accepted 15 December 2014

Academic Editor: Anindya Ghoshal

Copyright (c) 2015 Shangchen Fu et al. This is an open access article distributed under the Creative Commons Attribution License, which permits unrestricted use, distribution, and reproduction in any medium, provided the original work is properly cited.

For the purpose of improving the damage localization accuracy, a prewarping technology is combined with step pulse excitation and this method is used in Lamb wave imaging of plate structures with adjacent damages. Based on the step pulse excitation, various narrowband or burst response can be derived by signal processing technology and this method provides flexibility for further prewarping approach. A narrowband signal warped with a preselected distance is then designed, and the dispersion in the response of this prewarping signal will be greatly reduced. However, in order to calculate the distance for prewarping, the first arrival needs to be estimated from the burst response. From the step-pulse response, narrowband responses at different central frequencies can be obtained, and by averaging peak-value time of their first arrivals, a more accurate estimation can be calculated. By using the prewarping method to the damage scattering signals before imaging, the imaging resolution of the delay-and-sum method can be highly enhanced. The experiment carried out in an aluminum plate with adjacent damages proves the efficiency of this method.

\section{Introduction}

Lamb waves can travel over large distances with little energy loss and have high sensitivity to structural damages, which make them very suitable in structural health monitoring (SHM) to detect damages in plate-like structures [1-3]. A sparse matrix of transducers formed by piezoelectric wafers is enough to monitor a relatively large structure in SHM. Generally, damages are identified with certain imaging technology in Lamb wave defect detection [4-8]. Among all these imaging technologies, delay-and-sum algorithm is quite suitable for SHM imaging, because only a sparse PZT array is needed in this method.

In the delay-and-sum method, damage scattering signals obtained by subtracting the baselines from the damage signals are used for imaging. Although the signals of direct arrival and reflections from edges are removed in the damage scattering signals, imaging results are still greatly affected by the multimode and dispersion characteristic of Lamb waves, especially when there are adjacent damages.
By using low frequency burst wave as excitation, the number of Lamb wave modes can be reduced. Furthermore, with the frequency turning theory [9] or the double-side excitation method [10], one dominant mode can be selected. However, even for the single-mode signals of narrow band, the dispersive wave packets still spread out in time and space with their waveforms distorted as they propagate [11]. When there are adjacent damages, the wave packets from different damages will overlap with each other and the location of damages in the imaging results can hardly be identified. In order to improve the damage localization accuracy and enhance the spatial resolution of the imaging results, the dispersion effect must be suppressed.

The dispersion effect is caused by the nonideal transfer function of the propagation path; if we know this transfer function, we can use it reversely to compensate the dispersion. From the view point of signal selection, Lamb wave dispersion can be compensated by employing specific excitation signal. In this paper, a narrowband signal warped with a preselected distance is generated and applied as excitation 
in the detection. The warping function is derived from the wavenumber of the Lamb wave, and the dispersion in the response of this prewarping signal will be significantly reduced; even the wave packets from adjacent damages can also be clearly separated.

In order to calculate the distance for prewarping, the arrival time of the first peak in the damage scattering signal must be identified. Multifrequency responses and averaging method are used here to improve the estimation accuracy. According to the theory of signal and system, the response of arbitrary excitation signal can be calculated by the convolution of impulse response with this excitation. As the impulse response is the first-order derivative of step response, various narrowband responses including the response of the prewarping signal can all be derived from a one-time measurement of the step response. This technology brings much more flexibility in designing excitation signals and obtaining their responses. By combining this prewarping method with the delay-and-sum imaging method, the resolution of damage locations in Lamb wave imaging can be highly enhanced. Both the compensation result and the imaging result in an aluminum plate with adjacent damages show the efficiency of this method.

\section{Dispersion Compensation with Prewarping and Step Pulse Excitation}

2.1. Dispersion Effect of Lamb Waves. Assuming that there is only $A_{0}$ mode propagating in the structure, for a transducer pair $P_{A-B}$ composed of two PZT wafers, when $P_{A}$ is applied with a burst wave $v_{0}(t)$, the direct arrival received by $P_{B}$ in frequency domain can be described as

$$
V(f)=V_{0}(f) e^{-j k(f) d_{A B}},
$$

where $V_{0}(f)$ is the Fourier transform of $v_{0}(t), d_{A B}$ is the distance between $P_{A}$ and $P_{B}$, and $k(f)$ is the wavenumber of the structure. As the wavenumber changes nonlinearly with frequency, dispersion is generated, and it will become more serious when the propagation distance increases. Figure 1 shows the theoretical wavenumber of $A_{0}$ mode in an aluminum plate $1.5 \mathrm{~mm}$ thick. The nonlinearity in its low frequency part is particularly obvious.

In Lamb wave defect detection, damage scattering signal is usually used. Considering that there are two damages in the structure, and the propagation distances from $P_{A}$ to $P_{B}$ through the two damages are $d_{1}$ and $d_{2}$, respectively, then the main wave packets in the damage scattering signals can be expressed as

$$
V(f)=V_{0}(f) e^{-j k(f) d_{1}}+V_{0}(f) e^{-j k(f) d_{2}} .
$$

If $d_{1}$ and $d_{2}$ are close, the two wave packets will overlap together because of dispersion. Figure 2 shows the timedomain dispersive wave packets calculated from (2) and the inverse Fourier transform for a 3-cycle burst wave with the central frequency of $100 \mathrm{kHz}$ when $d_{1}$ and $d_{2}$ are $0.6 \mathrm{~m}$ and $0.7 \mathrm{~m}$, respectively. Due to the dispersion effect, the wave packets at the two propagation distances overlap with each

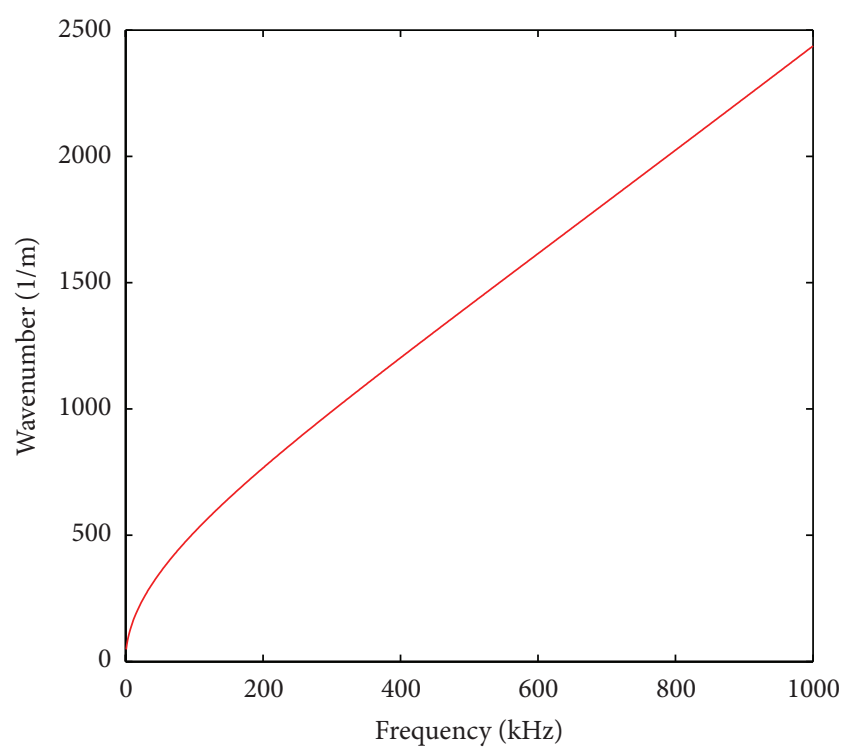

FIgURE 1: The wavenumber in an aluminum plate $1.5 \mathrm{~mm}$ thick.

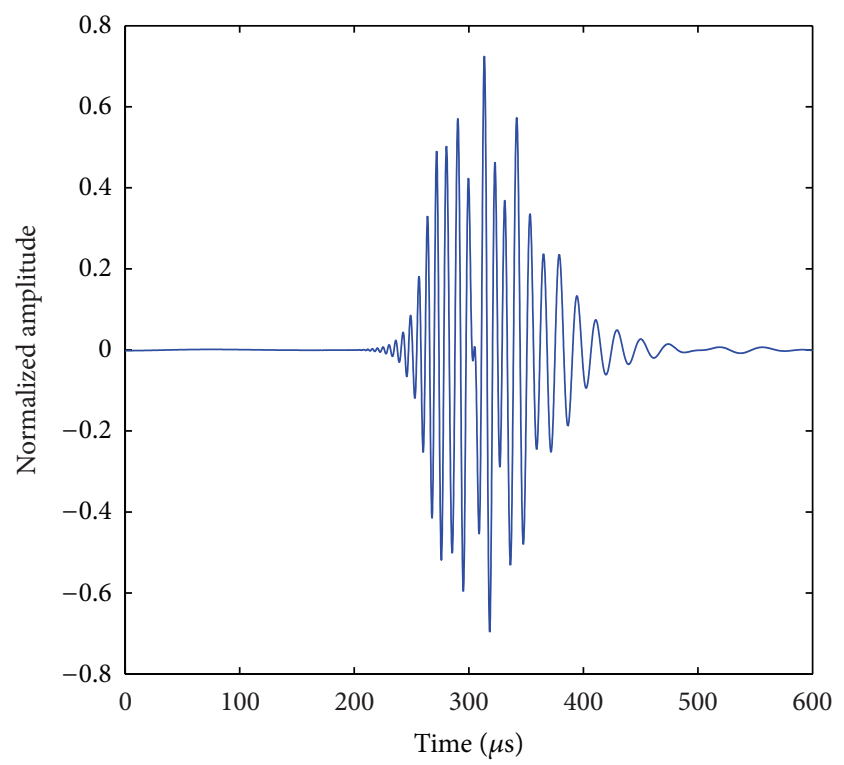

FIGURE 2: The sensing signal calculated with (2) for a $100 \mathrm{kHz} 3$-cycle burst wave.

other. The time-frequency representation of the wave packets is shown in Figure 3. We can see that frequency components of the two wave packets are warped with time and mixed together on the time-frequency plane because of dispersion. As the time-of-flight (TOF) of the wave packets cannot be extracted from the results in Figure 2 or Figure 3, the location of damages cannot be determined.

2.2. Dispersion Compensation with the Prewarping Method. We can see from (1) that two factors are contained in the sensing signal of Lamb wave, one is the excitation signal $V_{0}(f)$ and the other is the phase-delay factor $e^{-j k(f) d_{A B}}$. By removing 


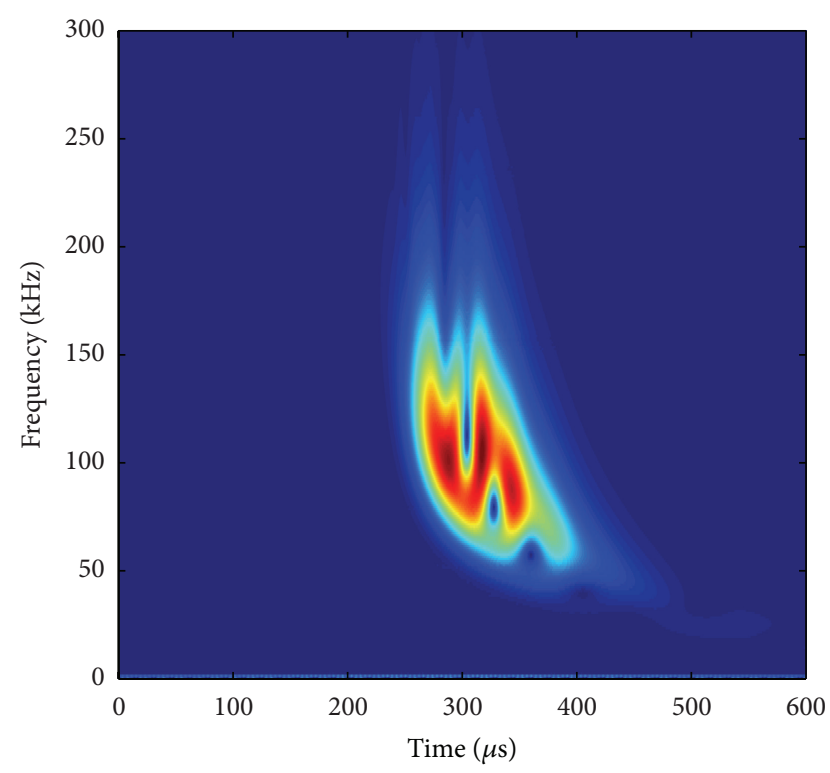

Figure 3: Time-frequency representation of wave packets in Figure 2.

the phase-delay factor, the dispersion can be eliminated. As the wavenumber $k(f)$ is determined by the structure, it is preferred to reduce the distance $d_{A B}$ to suppress the dispersion. Considering a signal which can be expressed as

$$
V_{0}^{\prime}(f)=V_{0}(f) e^{j k(f) d_{0}},
$$

then its response will be

$$
V^{\prime}(f)=V_{0}^{\prime}(f) e^{-j k(f) d_{A B}}=V_{0}(f) e^{-j k(f)\left(d_{A B}-d_{0}\right)} .
$$

The dispersion in (4) will be reduced when $d_{0}$ is growing and can be completely eliminated if $d_{0}$ is the same as $d_{A B}$. The result in (4) shows that the dispersion in the received signals can be suppressed by employing a prewarping signal as excitation.

When the prewarping signal is used as excitation, the extracted damage scattering signal will become

$$
\bar{V}(f)=V_{0}(f) e^{-j k(f)\left(d_{1}-d_{0}\right)}+V_{0}(f) e^{-j k(f)\left(d_{2}-d_{0}\right)} .
$$

The compensation effect will be greatly influenced by the preselected distance $d_{0}$. In the situation of adjacent damages, $d_{1}$ and $d_{2}$ are close, and the dispersion must be reduced as much as possible, so that the wave packets from different damages can be clearly separated. Practically, it is impossible to obtain the exact value of $d_{1}$ or $d_{2}$, but an approximate distance can be estimated from the extracted damage scattering signal in (2), as the main wave packets are from the damage area.

Figure 4 shows the envelope of the overlapped wave packets in Figure 2. The peak value of the envelope represents the most serious overlap part of the two damages, which can be used to estimate the distance for prewarping:

$$
d_{0}=c_{g} t_{0}
$$

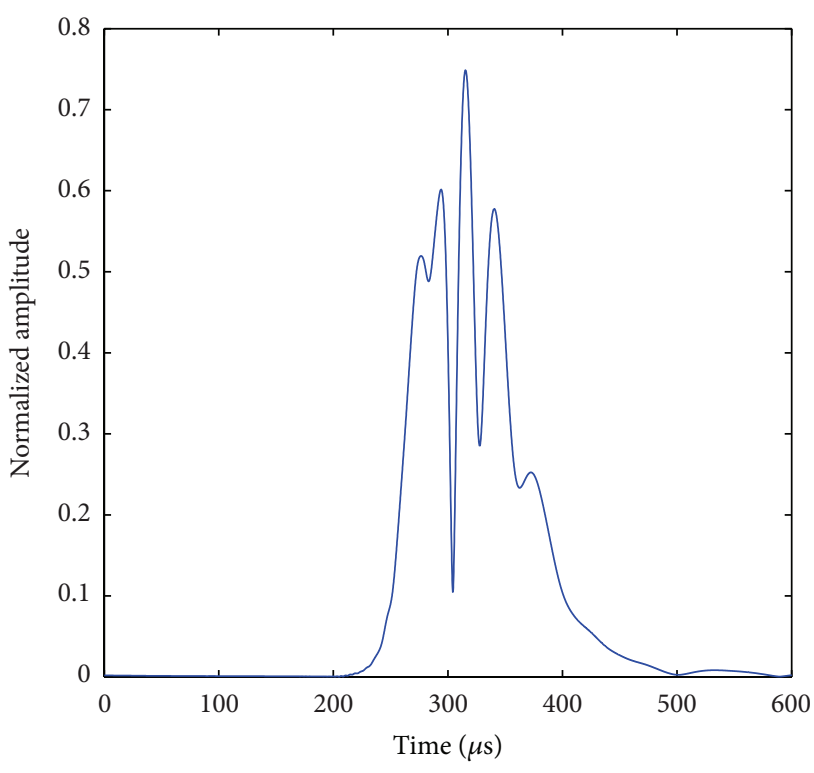

FIgURE 4: The envelope of the wave packets in Figure 2.

where $c_{g}$ is the group velocity at the central frequency of the narrowband excitation signal. The time of the peak value in Figure 4 is $315.3 \mu \mathrm{s}$, and the group velocity at the central frequency of $100 \mathrm{kHz}$ is $2190 \mathrm{~m} / \mathrm{s}$, so the preselected distance $d_{0}$ is about $0.69 \mathrm{~m}$. Then the prewarping signal can be generated with this distance, which is shown in Figure 5. The response of this prewarping signal calculated with (5) is shown in Figure 6, and the time-frequency representation is shown in Figure 7. Compared to the result in Figure 2, the dispersion in Figure 6 is significantly reduced, and the two wave packets can be clearly distinguished. The timefrequency characteristic in Figure 7 is greatly improved. From the compensated result in Figure 6, TOF can be extracted and be used to determine the exact location of damages.

2.3. Realization of the Prewarping Method with Step Pulse Excitation. From the above discussion we can see that both $V_{0}(f)$ and $V_{0}^{\prime}(f)$ are needed to be applied as excitation in the prewarping method, which is complicated and time consuming. In order to simplify the process, as well as to further improve the localization accuracy, step pulse is brought in and employed as excitation in the realization of the prewarping method.

According to the theory of signals and systems, the response of arbitrary signal $v_{0}(t)$ can be calculated with the impulse response $h(t)$ as

$$
v(t)=v_{0}(t) * h(t),
$$

where the asterisk represents the convolution operation. In frequency domain, (7) is transferred to

$$
V(f)=V_{0}(f) H(f)
$$




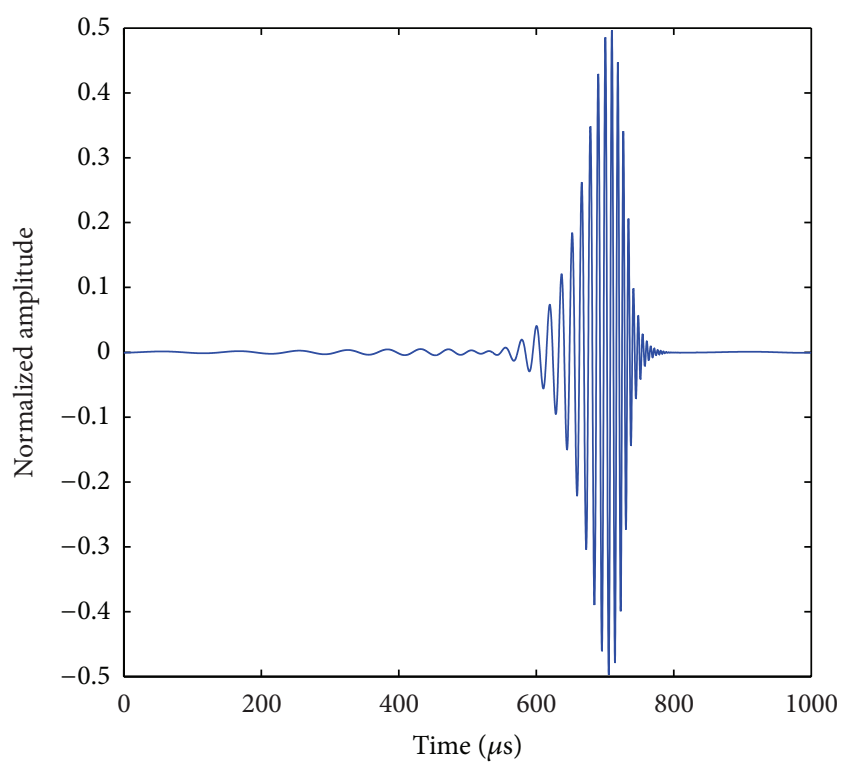

FIGURE 5: The prewarping signal with preselected distance of $0.69 \mathrm{~m}$.

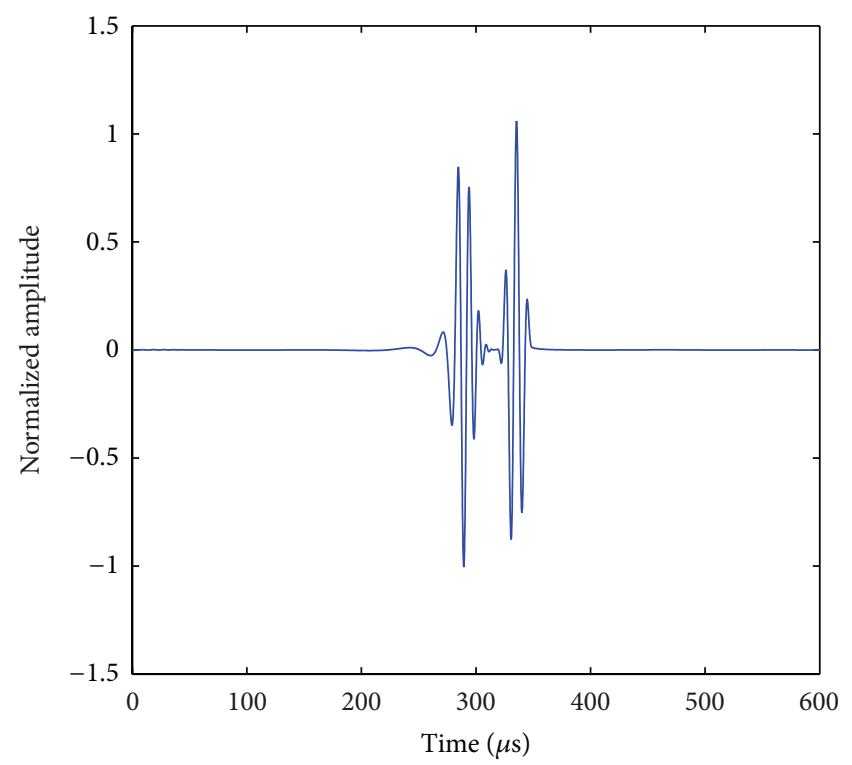

FIGURE 6: The response of the prewarping signal in Figure 5.

It is difficult to generate an impulse pulse with the signal generator, but the impulse response can be expressed as the first order derivative of the step-pulse response, which is

$$
h(t)=\frac{d[g(t)]}{d t},
$$

where $g(t)$ is the step-pulse response. From this point of view, step pulse can be applied as excitation to generate the impulse response.

Equation (8) shows that the response of both $V_{0}(f)$ and $V_{0}^{\prime}(f)$ can be obtained by calculation, and repetitive experiments can be avoided. Meanwhile, in the step of selecting the distance for prewarping, burst wave with different central

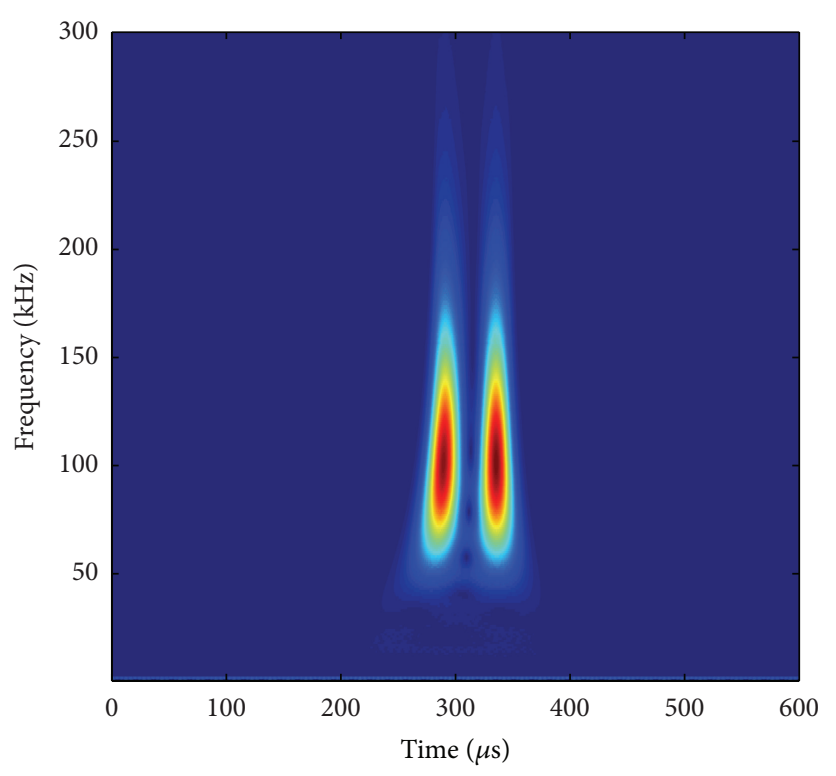

FIGURE 7: The time-frequency representation of the signal in Figure 6.

frequencies can be employed, and the following equation is used to extract the distance for prewarping:

$$
d_{0}=\frac{1}{n} \sum_{i=1}^{n} c_{g}\left[f_{c}(i)\right] t_{0}(i)
$$

where $n$ is the number of the selected central frequencies, $f_{c}(i)$ is $i$ th selected central frequency, and $c_{g}\left[f_{c}(i)\right]$ is the group velocity at this central frequency.

When step pulse is employed as excitation signal, the final compensated damage scattering signal in frequency domain can be expressed as

$$
V_{d s}(f)=V_{0}\left(f, f_{c}\right) e^{j k(f) d_{0}} H_{d h}(f),
$$

where $H_{d h}(f)$ is the Fourier transform of the broadband damage scattering signal $h_{d h}(t)$ :

$$
h_{d h}(t)=\frac{d\left[g_{d}(t)-g_{h}(t)\right]}{d t}
$$

where $g_{d}(t)$ and $g_{h}(t)$ are the step-pulse response in damaged and healthy mode, respectively. As both of $g_{d}(t)$ and $g_{h}(t)$ are measured by experiment, $h_{d h}(t)$ is also a measured signal.

According to (7), the time-domain signal of (11) can be expressed as

$$
v_{d s}(t)=v_{\text {pre }}\left(t, f_{c}\right) * h_{d h}(t)
$$

where $v_{\text {pre }}\left(t, f_{c}\right)$ is the time-domain prewarping signal, which can be obtained by

$$
v_{\text {pre }}\left(t, f_{c}\right)=\operatorname{IFT}\left[V_{0}\left(f, f_{c}\right) e^{j k(f) d_{0}}\right],
$$

where IFT $[\cdot]$ represents the inverse Fourier transform. From (13) we can see that only the step-pulse response needs to 
be measured in the realization of the prewarping method with step pulse excitation; the prewarping signal $v_{\text {pre }}\left(t, f_{c}\right)$ is just used for convolution, which means it is not necessary to be generated; an ideal one calculated by (14) would be appropriate. Besides, the central frequency $f_{c}$ of the burst wave $V_{0}\left(f, f_{c}\right)$ used in (14) can also be adjusted, which offers flexibility in selecting proper frequency for different structures.

In summary, the realization of the prewarping method with step pulse excitation needs three steps. Firstly, step pulse is applied as excitation in both healthy mode and damaged mode, and the broadband damage scattering signal $h_{d h}(t)$ is extracted from the received signals. Secondly, the response of the burst wave $V_{0}(f)$ is calculated with (7), and the distance for prewarping is estimated with (10); then the prewarping signal $v_{\text {pre }}\left(t, f_{c}\right)$ can be obtained. Lastly, the response of the prewarping signal is calculated with (13). The dispersion in the signal calculated with (13) will be significantly reduced and even the location of adjacent damages can be accurately identified.

\section{Modified Delay-and-Sum Imaging Method}

The delay-and-sum method is a simple and effective imaging method widely used in Lamb wave imaging. In this method, the detected structure is discretized to many grids, and each grid point is assumed as a reflection source. When an excitation signal is applied at a transducer $P_{m}\left(x_{m}, y_{m}\right)$, the signals received by $P_{n}\left(x_{n}, y_{n}\right)$ are supposed to be a superposition of signals reflected from all the discrete points on the structure. For a point $O(x, y)$, the traveling time of the path $P_{m}-O-P_{n}$ can be calculated by

$$
t_{m n}(x, y)=t_{\text {off }}+\frac{r_{m n}}{c_{g}},
$$

where $t_{\text {off }}$ is the reference time, $c_{g}$ is the group velocity at the central frequency of the narrow band signal, and $r_{m n}$ is the length of the propagation path $P_{m}-O-P_{n}$, which is

$$
\begin{aligned}
r_{m n}= & \sqrt{\left(x_{m}-x\right)^{2}+\left(y_{m}-y\right)^{2}} \\
& +\sqrt{\left(x_{n}-x\right)^{2}+\left(y_{n}-y\right)^{2}} .
\end{aligned}
$$

In Lamb wave defect detection, the envelope of the damage scattering signal is extracted at the first step. Then each point on the envelope is mapped to its corresponding grid in the structure according to (15) and (16). If the grid is a real flaw point, its value will be enhanced by superposition of the test results of several detection paths (with different $m$ and $n$ ); otherwise its value will be reduced by averaging, as is shown in Figure 8. The image value at grid point $O$ is determined by the following delay-and-sum method:

$$
G_{O}(x, y)=\left[\frac{2}{N(N-1)} \sum_{m=1}^{N} \sum_{n=m+1}^{N} v\left(t_{m n}(x, y)\right)\right]^{2},
$$

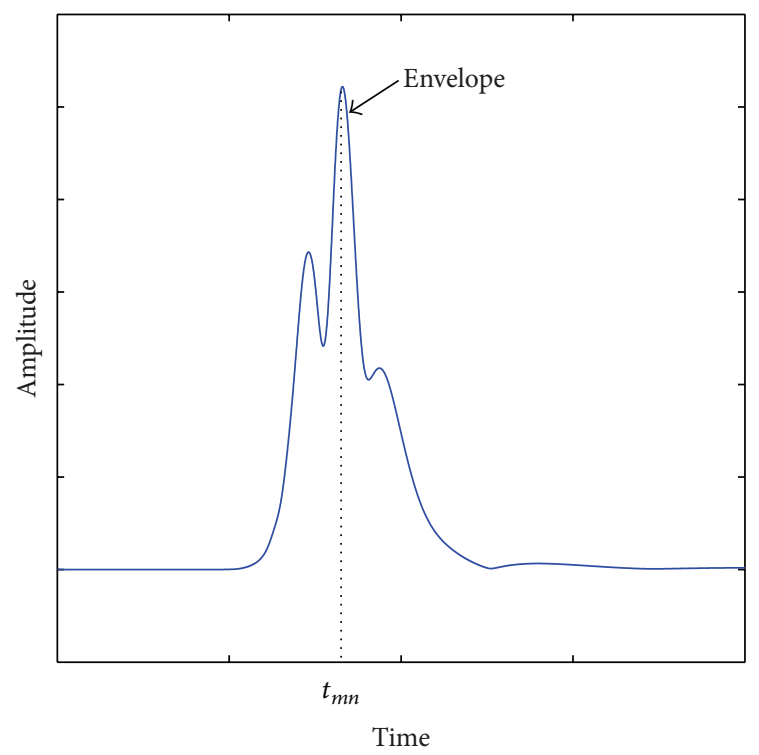

FIGURE 8: Illustration of delay-and-sum imaging.

where $v\left(t_{m n}\right)$ is the envelop of the damage scattering signal extracted from transducer pair $P_{m-n}$. The final image will automatically focus on the real damage point by using (17).

As has been pointed out, the dispersion effect will elongate the wave packets, and the imaging effect of traditional delay-and-sum method will be greatly influenced. In order to improve the resolution, the prewarping method can be employed before imaging. As the dispersion can be significantly reduced with the prewarping method, the imaging resolution can be highly improved. With the compensated damage scattering signal $v^{\prime}\left(t_{m n}(x, y)\right)$, the imaging value at $O$ will become

$$
G_{O}^{\prime}(x, y)=\left[\frac{2}{N(N-1)} \sum_{m=1}^{N} \sum_{n=m+1}^{N} v^{\prime}\left(t_{m n}(x, y)\right)\right]^{2} .
$$

\section{Experimental Verification}

4.1. Experimental Setup. An aluminum plate with the size of $1000 \mathrm{~mm} \times 1000 \mathrm{~mm} \times 1.5 \mathrm{~mm}$ is tested in experiment. Eight PZT wafers controlled by a switch matrix are mounted on the plate to form the detector array. The diameter of each PZT is $8 \mathrm{~mm}$ and its thickness is $0.48 \mathrm{~mm}$. Two identical hexagonal hollow screws with circumcircle diameter of $1 \mathrm{~cm}$ are bonded on the plate to simulate adjacent damages. The location of PZT wafers $\left(P_{1}-P_{8}\right)$ and the damages $\left(D_{1}, D_{2}\right)$ are shown in Figure 9. The coordinates of the two damages are $(10 \mathrm{~mm}$, $-90 \mathrm{~mm}$ ) and $(80 \mathrm{~mm},-30 \mathrm{~mm})$, respectively.

A step pulse with rise time of $0.25 \mu$ s shown in Figure 10 is produced by a LAI200-ISA arbitrary wave generator and amplified by a power amplifier to enlarge the monitoring area in the plate. According to the frequency turning theory, $A_{0}$ mode will be dominant when the central frequency is below $105 \mathrm{kHz}$, and the sensing signal can be treated as single-mode signal, so the burst waves with the central frequencies of $50 \mathrm{kHz}, 60 \mathrm{kHz}, 80 \mathrm{kHz}$, and $100 \mathrm{kHz}$ are selected to estimate 


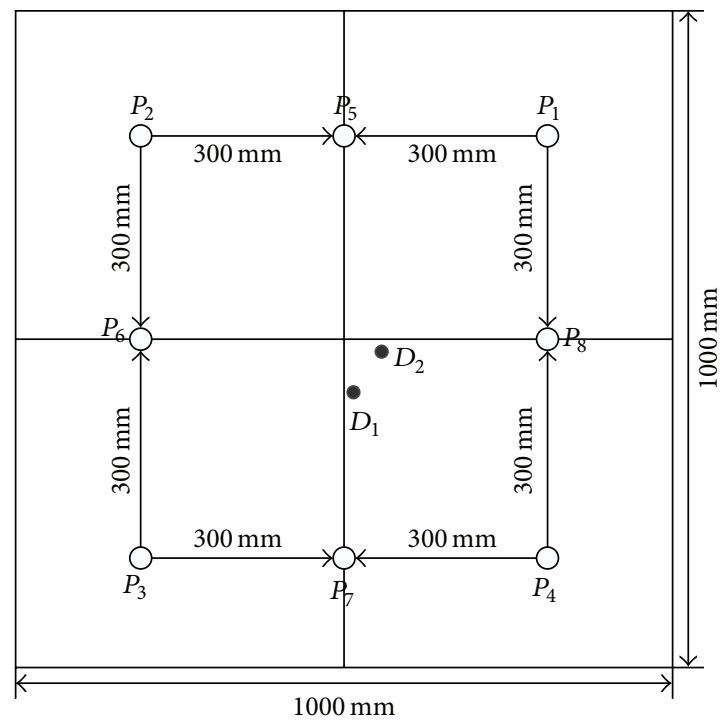

FIGURE 9: Configuration of the specimen.

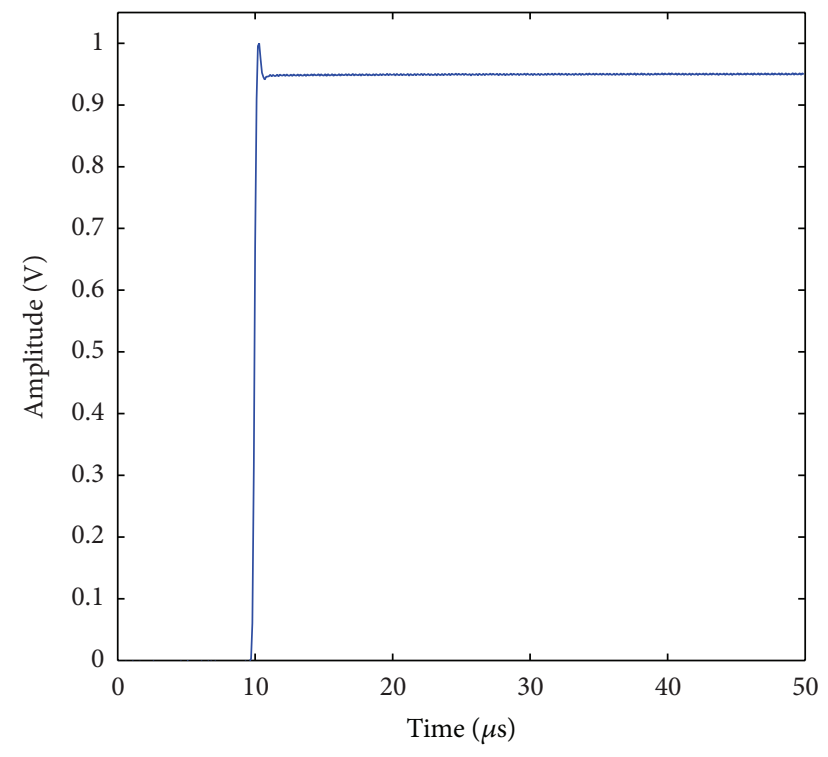

FIGURE 10: The step pulse excitation used in the experiment.

the distance for prewarping, and the burst wave with the central frequency of $100 \mathrm{kHz}$ is used to obtain the prewarping signal.

The frequency constant of the PZT wafer is $2000 \mathrm{~Hz} \cdot \mathrm{m}$, which means that its radial resonance frequency is about $250 \mathrm{kHz}$. Although the central frequencies used for obtaining the prewarping signal in the experiment are a little lower, they are still within the effective working range of the PZT wafer. Besides, both the excited and received signals are amplified with the amplifier to make sure that the collected signals are strong enough.

4.2. Compensation Effect of the Prewarping Method. The step pulse is applied at transducer $P_{1}$, and $P_{5}$ is selected to receive the response signals. Both healthy mode and damaged mode are tested. The broadband damage scattering signal calculated with (12) is shown in Figure 11(a). It can be converted to the response for the burst wave by (7). The envelopes of the calculated narrowband response at the selected central frequencies are shown in Figure 11(b). As the amplitude of all the envelopes between $650 \mathrm{~mm}$ and $900 \mathrm{~mm}$ is larger than other areas, the location of damages should be in this range. However, neither of the two damages can be exactly localized from the envelope because of dispersion. By selecting the max value at each central frequency, the distance for prewarping can be calculated with (10), which is $814.7 \mathrm{~mm}$ here. Then the prewarping signal with the central frequency of $100 \mathrm{kHz}$ is generated, and the result is shown in Figure 11(c). By calculating the convolution of the prewarping signal in Figure 11(c) with the broadband damage scattering signal in Figure 11(a), the compensated damage scattering signal can be obtained, which is shown in Figure 11(d). We can see that both of the two damages can be clearly identified in Figure 11(d), which means that the prewarping method can effectively compensate the dispersion and improve the localization accuracy of damages.

4.3. Imaging Results. Transducers $P_{1}$ to $P_{8}$ in Figure 9 are applied with the step pulse in turn, and the remaining transducers are used to receive signals each time. The damage scattering signals of all the transducer pairs are extracted and compensated with the prewarping method; then damage imaging results based on the traditional delay-and-sum method and the modified method can be obtained with (17) and (18), respectively, which are shown in Figure 12. " $X$ " shows the exact locations of damages.

Because of dispersion, the hot spots corresponding to the two damages in Figure 12(a) are extended and mixed to an unclear bright area. However, by using the prewarping method before imaging, the spatial resolution can be greatly improved. The two damages in Figure 12(b) can be clearly identified and the centers of the hot spots locate exactly 


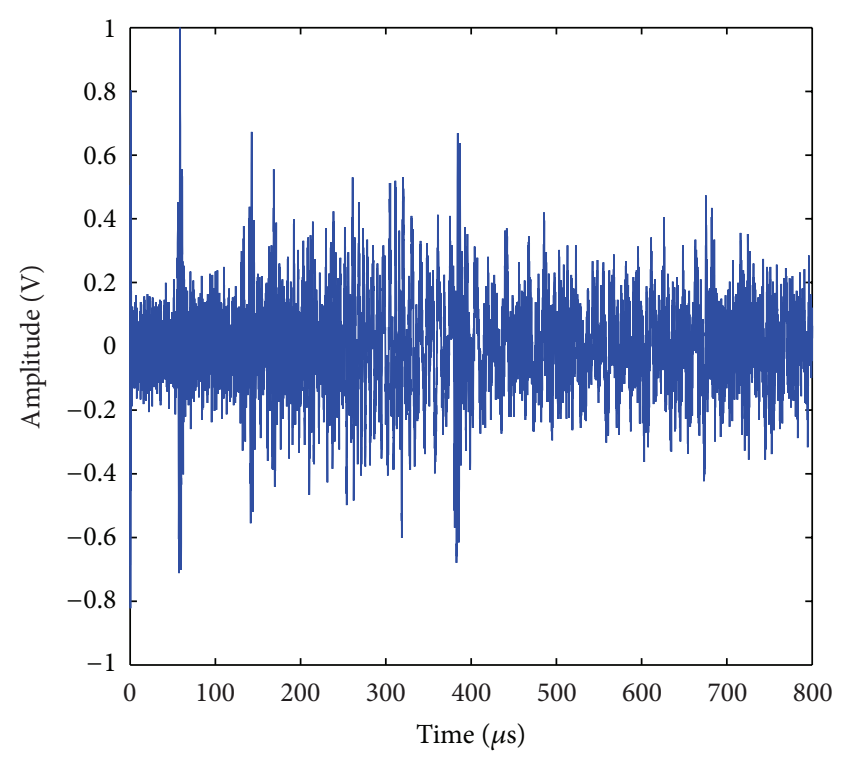

(a)

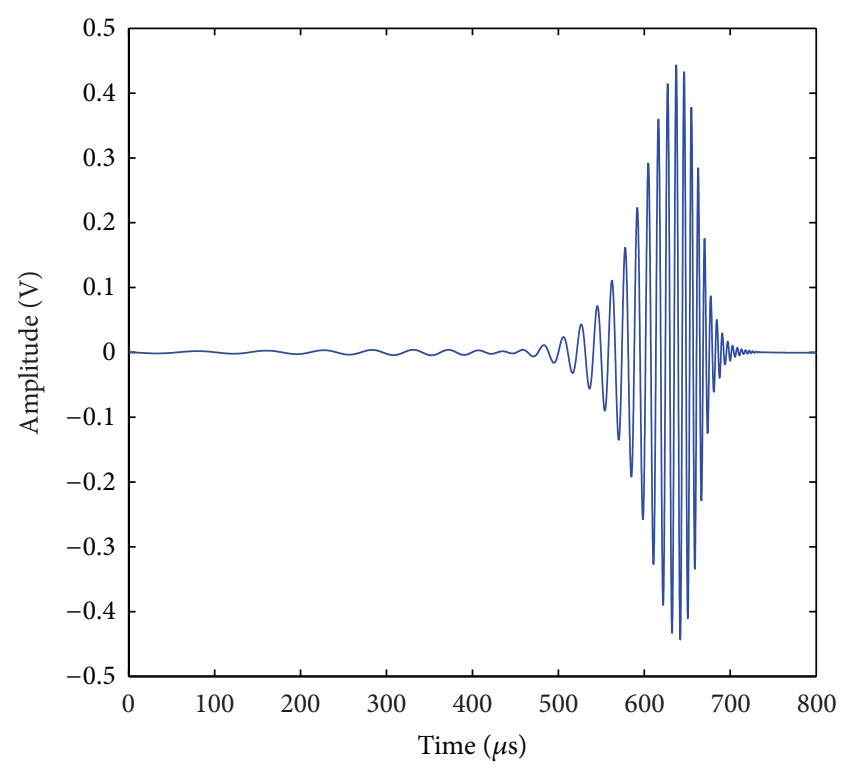

(c)

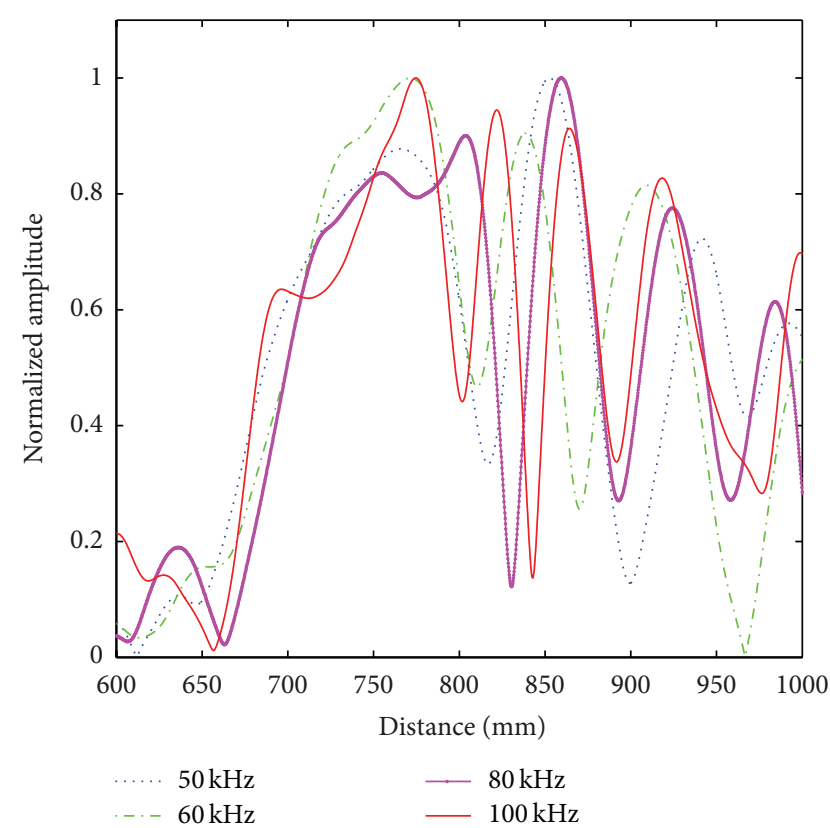

(b)

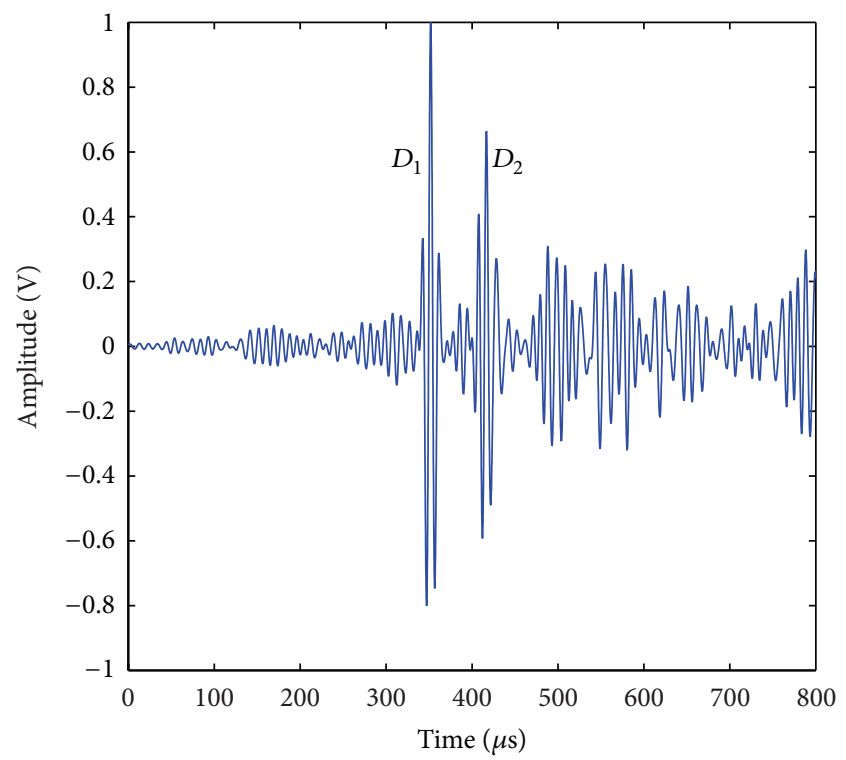

(d)

FIGURE 11: Compensation effect of the prewarping method. (a) The broadband damage scattering signal of $P_{1-5}$. (b) Envelopes of the response for burst wave at selected central frequencies. (c) The generated prewarping signal with the central frequency of $100 \mathrm{kHz}$. (d) Compensation result of the prewarping method.

at the real damage points. The imaging results in Figure 12 show that the prewarping method can significantly improve the damage localization accuracy and enhance the damage imaging resolution.

\section{Conclusion}

A prewarping method is proposed to improve the damage localization accuracy in Lamb wave imaging. A narrowband signal warped with a preselected distance is generated; the dispersion in the response of this prewarping signal will be greatly reduced after propagation in the tested structure. In order to simplify the process, step pulse excitation is selected, and all the narrowband responses used in the prewarping method can be obtained from the step-pulse response by convolution. By using prewarping method to the damage scattering signals before imaging, the imaging resolution of the delay-and-sum method can be highly enhanced. The experiment carried out on an aluminum plate with adjacent damages shows the efficiency of this method. 


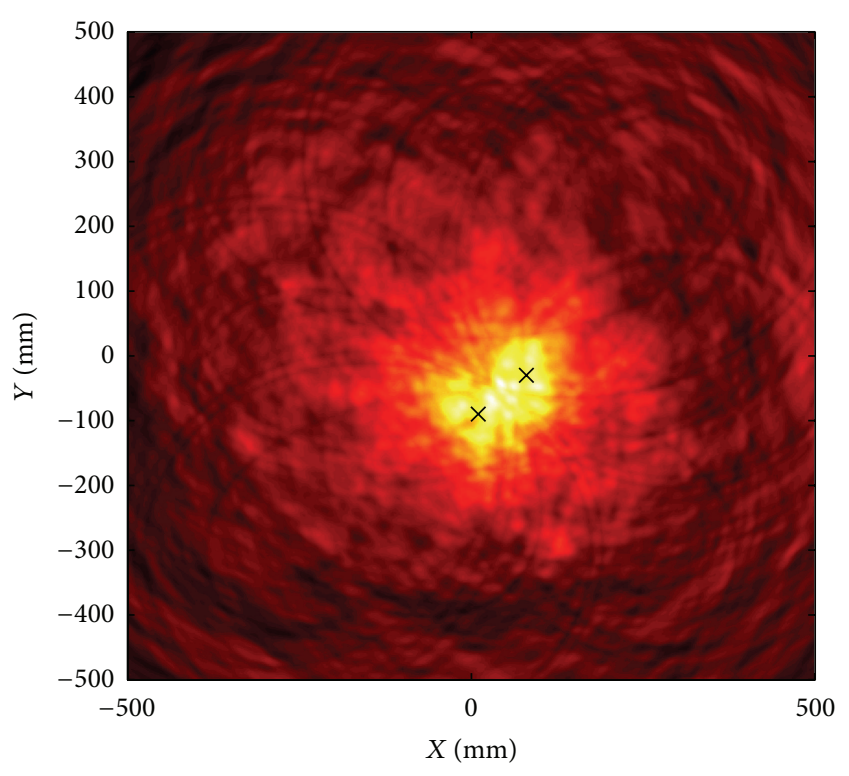

(a)

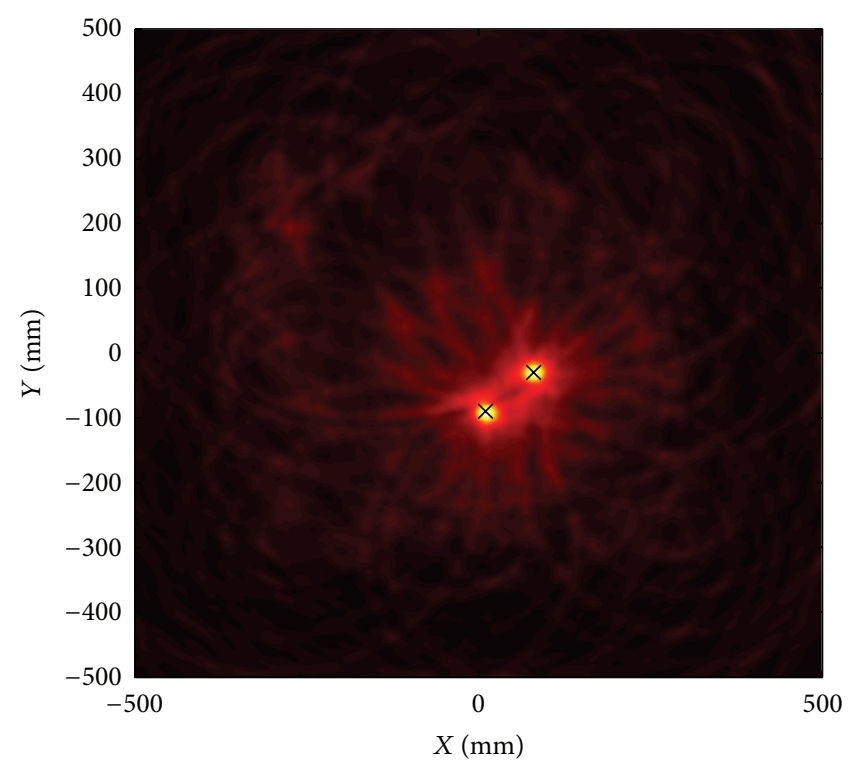

(b)

FIGURE 12: Imaging results. (a) Imaging result of the traditional delay-and-sum method. (b) Imaging result of the modified delay-and-sum method.

\section{Conflict of Interests}

The authors declare that there is no conflict of interests regarding the publication of this paper.

\section{Acknowledgment}

This study was supported by Natural Science Foundation of China (Grants nos. 51477183 and 51407198).

\section{References}

[1] B. Rocha, C. Silva, and A. Suleman, "Structural health monitoring system using piezoelectric networks with tuned lamb waves," Shock and Vibration, vol. 17, no. 4-5, pp. 677-695, 2010.

[2] L. Wang and F. G. Yuan, "Active damage localization technique based on energy propagation of Lamb waves," Smart Structures and Systems, vol. 3, no. 2, pp. 201-217, 2007.

[3] X. Qing, A. Kumar, C. Zhang, I. F. Gonzalez, G. Guo, and F.-K. Chang, "A hybrid piezoelectric/fiber optic diagnostic system for structural health monitoring," Smart Materials and Structures, vol. 14, no. 3, pp. S98-S103, 2005.

[4] R. Sicard, J. Goyette, and D. Zellouf, "A SAFT algorithm for lamb wave imaging of isotropic plate-like structures," Ultrasonics, vol. 39, no. 7, pp. 487-494, 2002.

[5] K. R. Leonard, E. V. Malyarenko, and M. K. Hinders, "Ultrasonic Lamb wave tomography," Inverse Problems, vol. 18, no. 6, pp. 1795-1808, 2002.

[6] C. J. Keulen, M. Yildiz, and A. Suleman, "Damage detection of composite plates by Lamb wave ultrasonic tomography with a sparse hexagonal network using damage progression trends," Shock and Vibration, vol. 2014, Article ID 949671, 8 pages, 2014.

[7] J. E. Michaels and T. E. Michaels, "Guided wave signal processing and image fusion for in situ damage localization in plates," Wave Motion, vol. 44, no. 6, pp. 482-492, 2007.
[8] J. E. Michaels, "Detection, localization and characterization of damage in plates with an in situ array of spatially distributed ultrasonic sensors," Smart Materials and Structures, vol. 17, no. 3, Article ID 035035, 2008.

[9] B. Xu and V. Giurgiutiu, "Single mode tuning effects on lamb wave time reversal with piezoelectric wafer active sensors for structural health monitoring," Journal of Nondestructive Evaluation, vol. 26, no. 2-4, pp. 123-134, 2007.

[10] J. Cai, S. Yuan, X. Zhang, and Q. Wang, "Lamb wave double side excitation and its application in adjacent damages monitoring," Journal of Nanjing University of Aeronautics and Astronautics, vol. 42, no. 1, pp. 62-67, 2010.

[11] P. Wilcox, M. Lowe, and P. Cawley, "Effect of dispersion on longrange inspection using ultrasonic guided waves," NDT and E International, vol. 34, no. 1, pp. 1-9, 2001. 

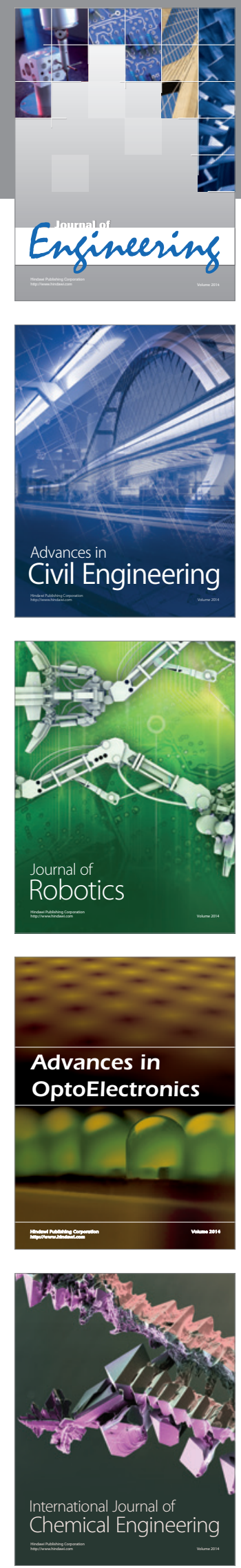

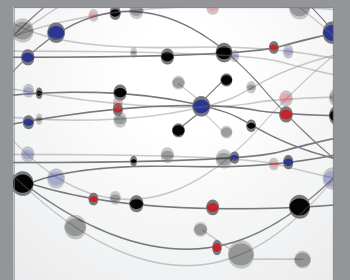

The Scientific World Journal
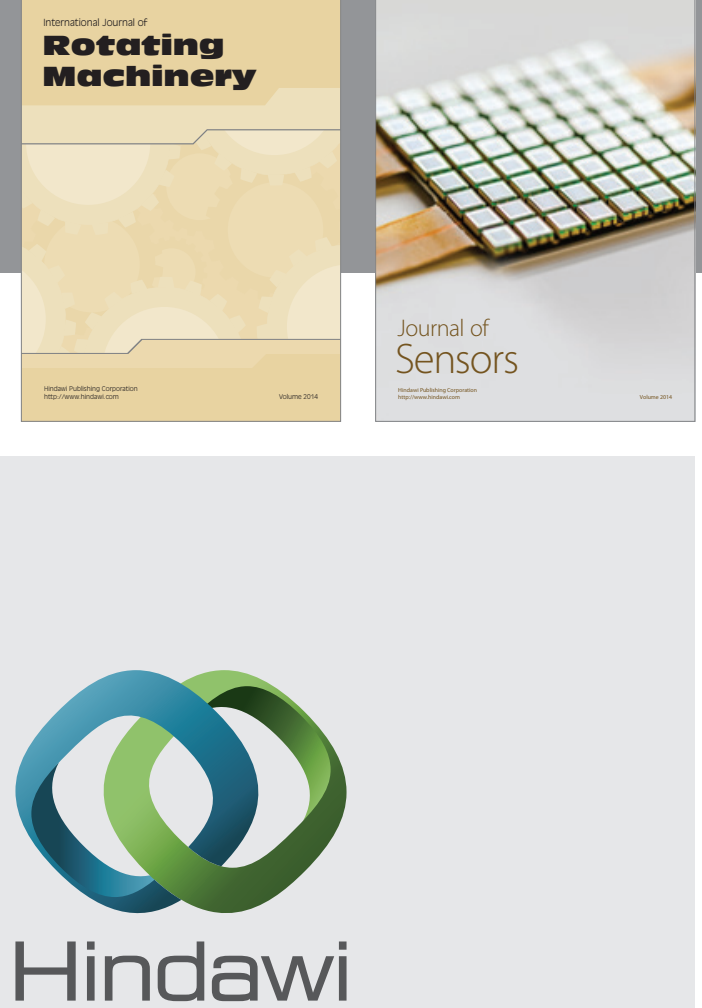

Submit your manuscripts at http://www.hindawi.com
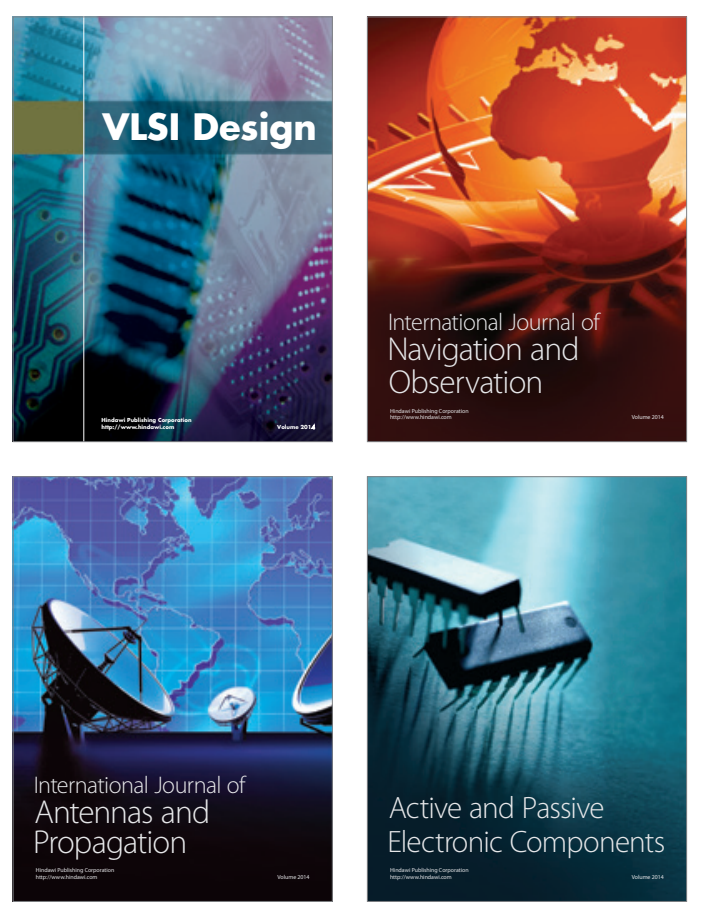
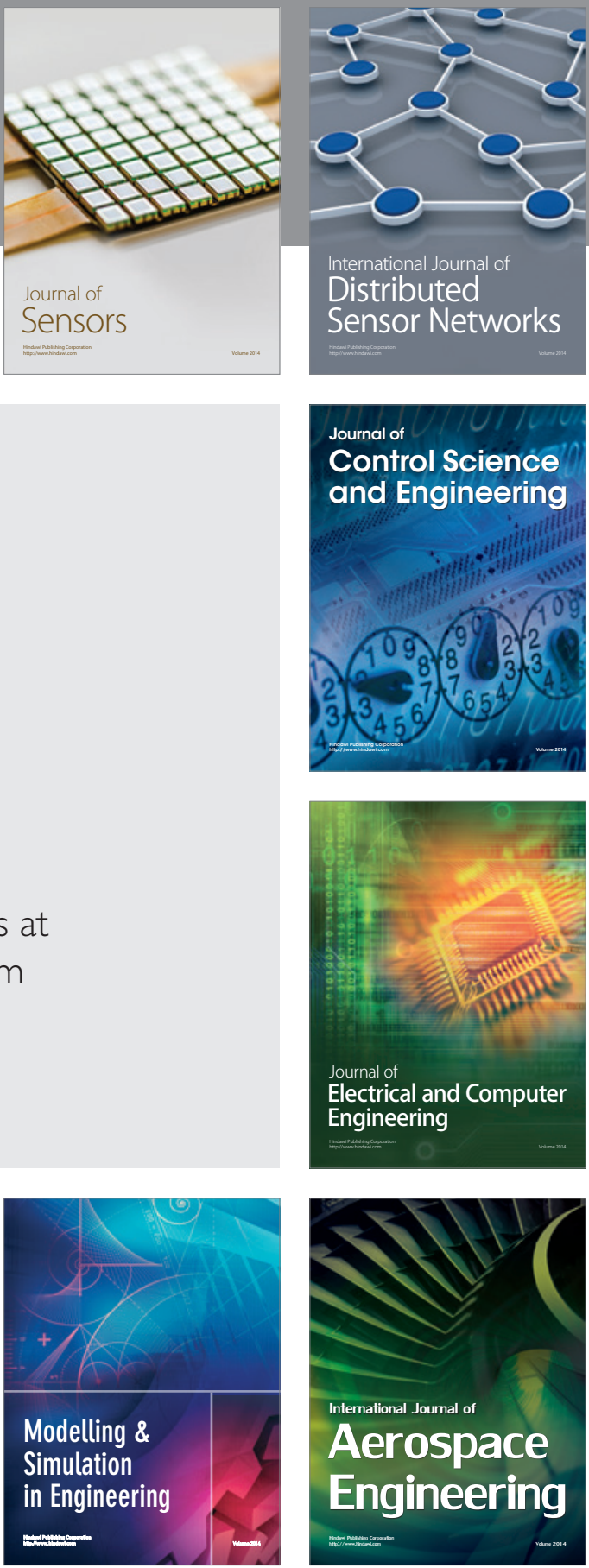

Journal of

Control Science

and Engineering
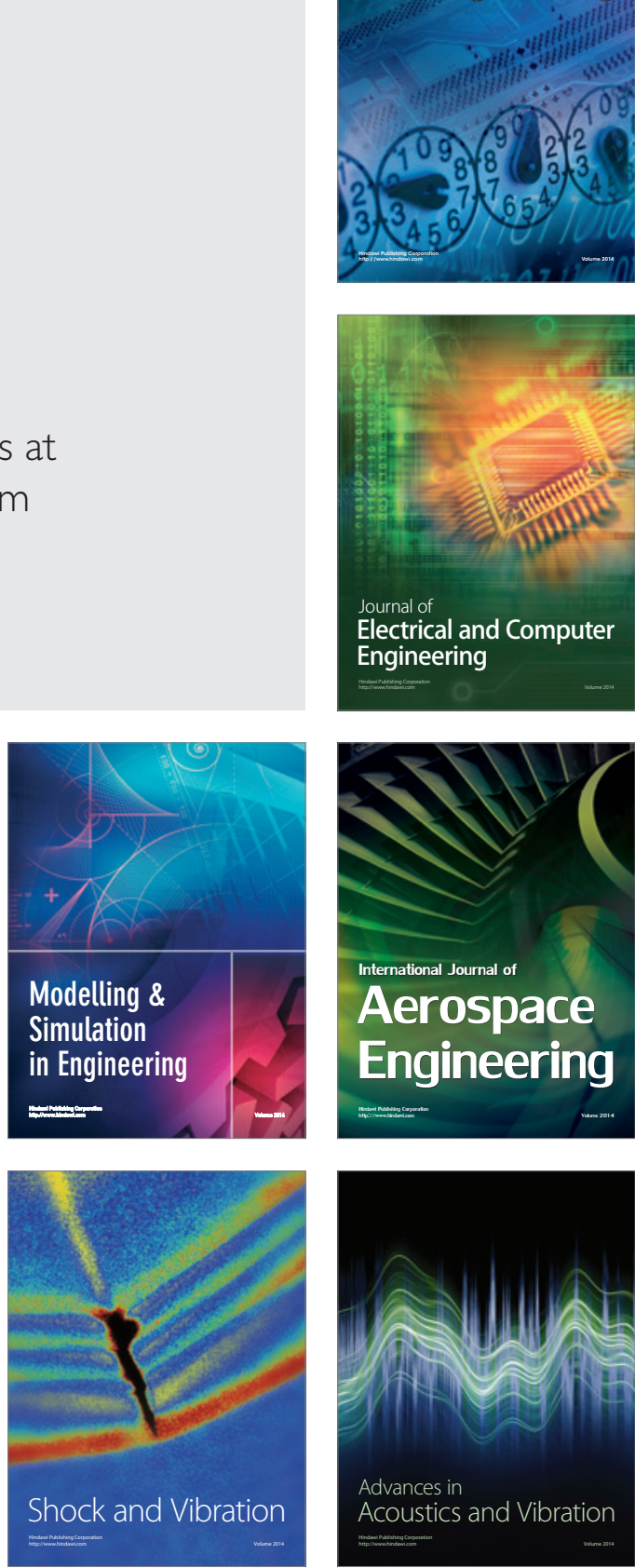